\title{
Consecutive photocatalysis vs electro-photocatalysis. Excitation of radical anions of naphthalene diimides.
}

\author{
Sofia Caby, Lydia M. Bouchet, Juan E. Argüello, Roberto A. Rossi and Javier Bardagi* \\ INFIQC, Departamento de Química Orgánica, Facultad de Ciencias Químicas, Universidad Nacional de \\ Córdoba, X5000HUA Córdoba, Argentina. E-mail: jibardagi@unc.edu.ar
}

\begin{abstract}
Photo- and electrochemical transformations represent excellent opportunities for the development of methodologies environmentally friendly and combination of both techniques would result in improved methodologies. Recently, photochemical transformations involving the excitation of open-shell species has become a reality. Photostimulated electron transfer (PET) from radical anions of organic dyes have been used in consecutive photoredox catalysis and electro-photocatalysis transformations, allowing the conversion of aryl halides in valuable compounds under mild conditions. The similarities of both techniques raise the question of the convenience of using one or other related and a close evaluation is necessary to drive further development and have been shyly done in previous works. However, an objective comparison of both approaches will require using the same chemical system. We present here a direct comparison of a "pure" photochemical and an electro-photochemical transformartion studying a model transformation, namely, a $\mathrm{C}-\mathrm{H}$ substitution in (hetero)aromatic systems with a C-C bond formation through reaction of halogenated substrates. Through a detailed investigation of both mechanisms, we identify a system that could be applied in both approaches with minor (necessaries) changes in the reaction conditions and clearly identify the excitation of radical anion of naphthalene diimides as the key intermediates. Both approaches are comparable in yields and kinetics with slightly better yields for the consecutive PET reaction and better selectivity for the electro-PET process. Our work offers a system that could be used for a more profound comparison of the experimental setups which could include, for instance, the important factor of energy consumption.
\end{abstract}

Keywords: photoredox catalysis, conPET, photo-EC', electro-photocatalysis 



\section{Introduction}

Photo- and electrochemical transformations represent excellent opportunities for the development of methodologies environmentally friendly, light and electrons are clean and cheap reagents without residual waste. In particular, advances in photoredox catalysis with visible light and mediated electrosynthesis have shown the versatility of these approximations in the synthesis of organic compounds. ${ }^{1-7}$ A close look at the mechanism of both approaches rapidly highlights shared key intermediates radical ions as a potential point of combination to design improved methodologies (Scheme 1).

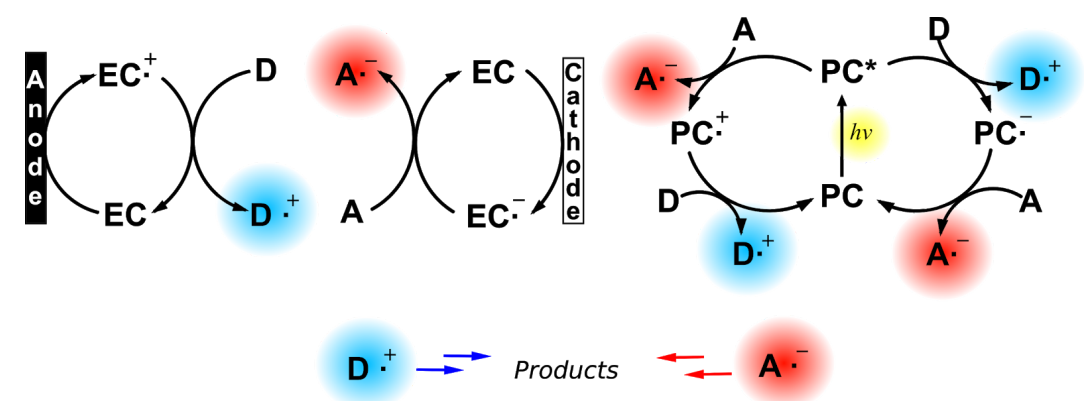

Scheme 1. Typical mechanism of mediated electrochemistry and photoredox catalysis.

(EC=electrocatalyst, $\mathrm{PC}=$ photocatalyst $)$

Approaches following a photoredox process require an electron transfer (ET) process from the excited state of the photocatalyst. Consequently, in terms of kinetic it is desired this deactivation path to be the main or comparable to others, such as fluorescence or non-radiative deactivation. Quenching of excited states of closed-shell organic molecules have been thoroughly used in photocatalytic synthetic transformations and the factors that dominate the mechanism of deactivation have also received important attention. On the other hand, excitation of open-shell organic species have recently appeared as a real synthetic useful method. In 2014, König et al. proposed the excitation of an organic radical anion specie to promote an interesting metal-free C-H substitution reaction (Scheme 2) ${ }^{10}$, in this case excitation of a perylene diimide (PDI) radical anion, an open-shell specie, makes possible the reduction of Ar-X by visible light and stable organic dyes as catalysts. Although electron transfer from excited radical anions could be tracked to $1970 \mathrm{~s},{ }^{11,12}$ recent works demonstrated the synthetic potential of the quenching of excited open-shell organic species in promoting challenging transformations under mild conditions. ${ }^{10,13-16}$
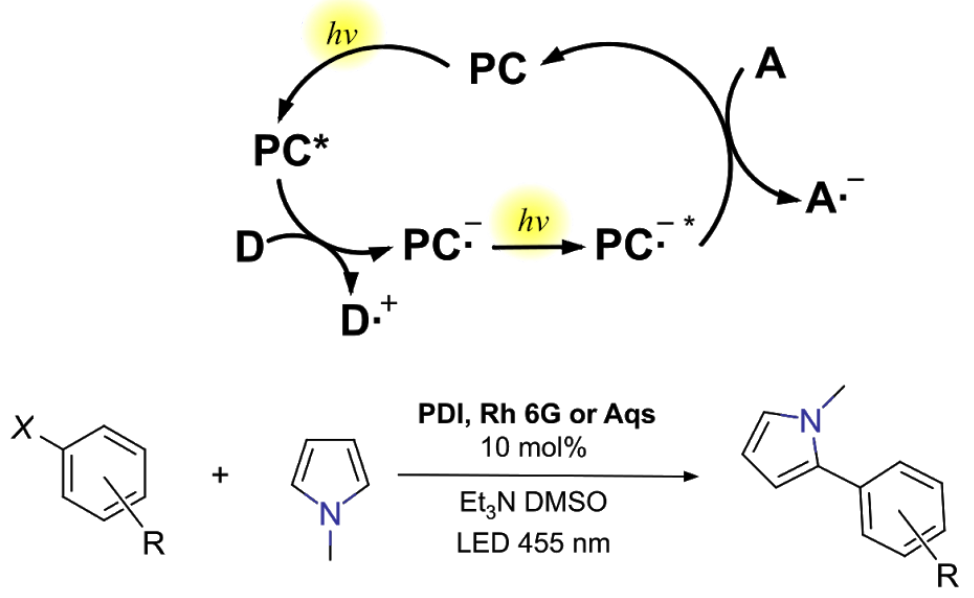

Scheme 2. Consecutive photoinduced electron transfer (conPET).

Previously to König's work, it was well established that perylene diimides (PDI) upon photoexcitation generates radical anions (PDI•-) in the presence of suitable sacrificial electron donors such as trialkylamines. These persistent radical anions absorb visible light and could be kept for hours in oxygen free atmospheres. 

Based on these characteristics, König and co-workers propose two consecutive PET (conPET) to access synthetic useful aryl radicals from aryl halides, through the strong reducing excited state of PDI - (PDI $\left.\bullet-^{*}\right)$ generated by two subsequent absorption of visible light photons starting from air-stable PDI (Scheme 2). Biologically relevant pyrrole derivatives were used as trapping of aryl radicals and functionalized pyrroles were synthesized in good to excellent yields by a $\mathrm{C}-\mathrm{H}$ substitution. An extension of this work used bay-substituted PDIs. ${ }^{17-19}$ Additionally, the conPET concept has been also used in ATRA reactions such as iodoperfluoroalkylation of alkenes. ${ }^{20}$

Mechanistic studies performed confirmed photo-stimulated formation of PDI •- in the presence of triethylamine through spectroscopy studies (UV-Vis and quenching of fluorescence). The needed for photostimulation of $\mathrm{PDI} \bullet-$ to promote ET to the substrates was confirmed by dark-light experiments in the presence of substrate of a chemical obtained solution of PDI $\bullet{ }^{10}$ (similar experiment was performed with supported PDIs. ${ }^{18}$ After ET to aryl halides the photo-induced transformation proceed via a radical mechanism, as probed using a radical clock precursor and trapping experiment with 2,2,6,6-tetramethylpiperidinoxyl (TEMPO). ${ }^{10}$ A recent picosecond time-resolved transient absorption (TA) spectroscopy of PDI - * confirmed, based on a direct observation, PET from this excited state to aryl halides, taking any doubt about this step sometimes questioned due to the relative short lifetime reported for radical anions of PDI (139 ps). ${ }^{21}$

The conPET catalytic cycle is not restricted to PDI derivatives and well known Rhodamine 6G dye (Rh 6G) demonstrated to be an excellent photocatalyst for this purpose enabling catalytic transformations of (hetero)aryl-halide bonds in interesting $\mathrm{C}-\mathrm{H}$ arylation reactions which involve the activity of $\mathrm{Rh} 6 \mathrm{G} \bullet \boldsymbol{*}^{*}{ }^{13,22}$ Another group of compounds known to form persistent radical anions are the quinone family (Figure 1). In particular 9,10-anthraquinones (Aqs) and their derivatives form coloured radical anions upon single-electron reduction. The radical anions of Aqs (Aqs $\left.{ }^{-}\right)$possess moderate reduction potentials $(-0,8--0,5 \mathrm{~V}$ vs. SCE) but Lund and co-workers ${ }^{23}$ already showed in 1985 that their excited states can transfer electrons to organic substrates such as 1,2-dibromobenzene, an aryl halide substrate with a reduction potential of $-1.88 \mathrm{~V}$ (vs. SCE). Aq and AqOH (Figure 1) were able to catalyse the reaction described in Scheme 2 in similar conditions, through a multiple photon catalytic cycle similar to the one described for PDI and Rh $\mathbf{6 G}$. However, in the case of Aqs a more complex picture is operative in which a semiquinone anion (AqsH-) formed from the Aqs $\bullet$ - (by proton and electron transfer) is responsible for the main transformation. ${ }^{24}$

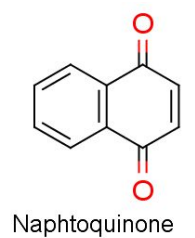

Figure 1.
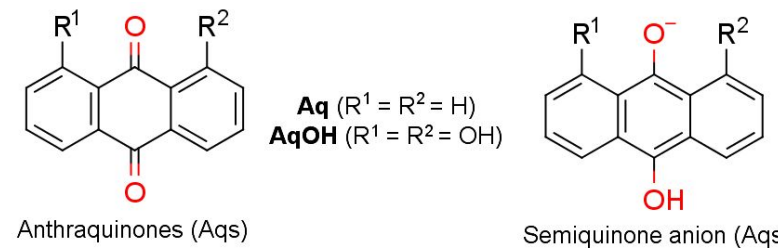

Semiquinone anion (Aqs $\mathrm{H}-)$

Structure of quinones and semiquinone anions.

Lund and Eriksen studied the photochemical behaviour of a electrochemically generated solution of Aq•and demonstrated a PET to aryl halides from this radical anion unequivocally, ${ }^{23}$ the difference in the photocatalytic transformation is the short life time of Aqs •- when prepared by ET from trialkylamines, since the ion pair evolve to the semiquinone radical and finally to AqsH-.

Further studies of electrochemically prepared radical anions of quinones derivatives (including AqOH, Figure 1) were performed by Eggins and Robertson. The author included in these cases aryl halides and carbonyl derivatives such as ketones, aldehydes, and glyoxylic acid as electron acceptors and probed the PET identifying products from the radical anions of these compounds. Results supported a general behaviour for a PET from doublet states quinones radical anions. ${ }^{25}$

PET from open-shell species have been observed in cyclic voltammetry experiments for other radical anions species including the ones from pyrene, ${ }^{12,26}$ perylene, ${ }^{12}$ anthracene, 9,10-dicyanoanthracene, dimethyl terephthalate ${ }^{26}$ and tetrachlorobenzoquinone. ${ }^{27}$ The experiments are consistent with a photo-EC' mechanism 

as Scheme 3 shows. ${ }^{27,28}$

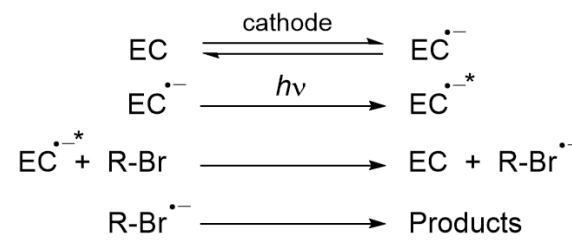

Scheme 3. Reductive Photo-EC' mechanism.

A reductive photo-EC' and the conPET mechanisms share a key step involving a radical anion excitation and it could be recognized that an electro-photocatalytic transformation could offer an alternative synthetic approach, based on the success of the conPET approach. An electrochemically generated radical ion implies replacing a stoichiometric amount of a sacrificial donor by a cathode, a situation that could be very advantageous having an easily tunable source of electrons. This was recognized by Lambert's and Wickens' groups between others who recently developed synthetic transformation using radicals anions and radicals cations. $^{15,16,29}$

Using dicyanoanthracene (DCA) as catalyst Lambert and Lin et $\mathrm{a}^{15}$ were able to modify aryl chlorides bearing electron-withdrawing and more importantly electro-donor substituents (Scheme 4.). Interesting borylation was achieved with broad toleration of functional groups such as ester, ketone, amide, carbamate, thiophene, and $\mathrm{N}$-Boc indole. Besides extention to $\mathrm{C}-\mathrm{H}$ funtionalization of (hetero)aromatic was possible but less explored. A photo-EC' mechanism was proposed without detailed studies. Key PET from the excited DCA- to aryl halides generated an aryl radical that react with different trapping agents $\left(\mathrm{B}_{2} \mathrm{pin}_{2}, \mathrm{Sn}_{2} \mathrm{Me}_{6}\right.$ and (Het)arene) to give the final product. Almost simultaneously, Wickens and co-worker ${ }^{16}$ developed a similar approach using naphtalenemonoimide NpMI as catalyst and performed phosphorylation and (hetero)arylation (C-H substitution) of aryl chlorides with moderate to good yields. EWG and EDG were tolerated allowing the reaction of substrates with potential as low as $-3.4 \mathrm{~V}$ (vs. SCE). The advantage of using a combination of techniques instead of pure electro- or photocatalysis was demonstrated in comparative experiments. These experiments showed the unique performance of the combination especially in substrates with highly negative $\mathrm{E}^{\mathrm{o}}$.

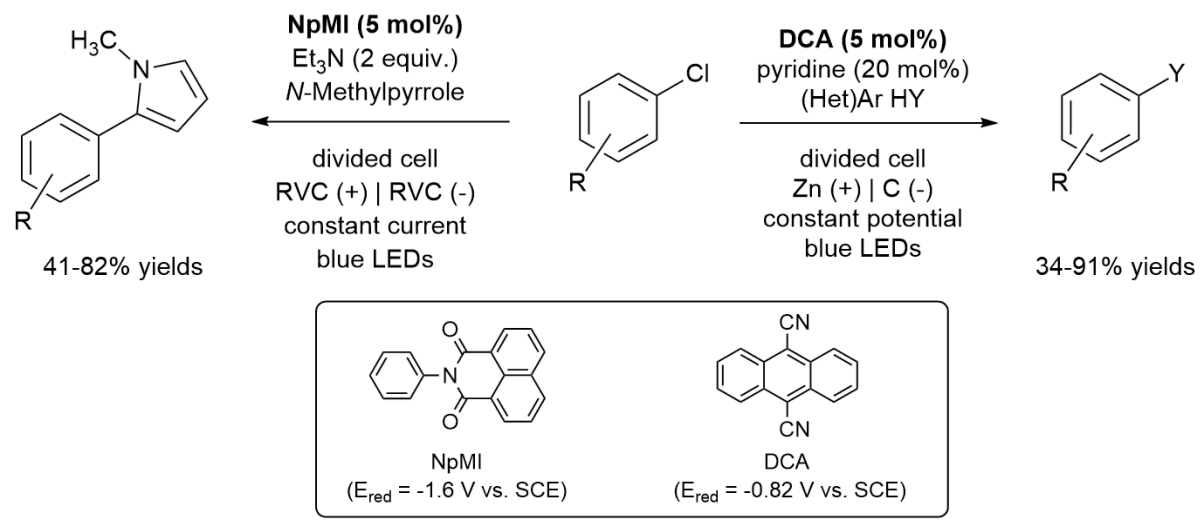

Scheme 4. Electrophotocatalyitc funtionalization of aromatic halides.

Despite these and other important contributions, ${ }^{9,30,31}$ approaches focused on the excited state of the open-shell organic molecules (radical or radical ions) are still unexplored in comparison with the "classical" photostimulated approach. Besides electro-photocatalytic works did not cover all the potential advantages of the electro-photocatalytic combination in terms of reducing the stoichiometry additives because they still use sacrificial donors ${ }^{16}$ or sacrificial anodes ${ }^{15}$ to achieve useful synthetic results.

Inside our continuous efforts to develop new catalytic systems, we identify naphthalene diimides (NDI) as potential candidates for demanding transformation inside visible light photocatalysis. Particularly, its use in a 

conPET approach and electro-photocatalysis would broad the applicability of these privileged structures used in other catalysis such as ion- $\pi$ catalysis and applications in supramolecular chemistry, sensors, host-guest complexes for molecular switching devices, ion-channels, intercalations with DNA for medicinal applications, etc. ${ }^{32}$ ET form excited NDI radical anions have been reported in laser flash photolysis experiments $^{33}$ and more recently, a conPET mechanism have been proposed with a NDI photocatalyst as part of a complex Metal-Organic Framework heterogeneous catalys ${ }^{34}$ although ET to aryl halides was successful, a constructive approach has not been explored and only dehalogenation was reported. The same material was probed in other catalytic activities and an important reaction of oxiranes with $\mathrm{CO}_{2}$ was accomplished with good results, showing the potential of this core in the construction of polyfunctional catalyst.

Based on the interesting potential shown in the previously described electro-photocatalytic transformations we wonder how it could be better to evaluate the advantages compared to "pure" photocatalyzed reactions. The differences found in the anthraquinone case comparing a conPET approach and an electrochemical PET, highlights a need for more mechanistic studies of similar systems, in order to recognize the general characteristics responsible for this kind of behavior that appears with open-shell species in similar conditions. Besides, mechanistic analysis in conditions closer to the preparative organic synthesis are increasingly demanding in addition to classical spectroscopies analysis. We believe an integral comparison will be beneficial for the future development of the combination and in doing so using the same chemical system would be favorable.

We show here our contribution to this final goal identifying a system that could be applied in both approaches with minor changes in the reaction mechanism and conditions. We study as a model transformation a C-H substitution in (hetero)aromatic systems with a $\mathrm{C}-\mathrm{C}$ bond formation through reaction of halogenated substrates. Using naphthalene diimides as photocatalysts, we develop and compare a "pure" photocatalytic and an electro-photocatalytic approach through a detailed investigation of both mechanisms.

\section{Results and Discussion}

\section{Synthesis and characterization of naphthalene diimides catalysts (NDIs)}

As mentioned before, naphthalene diimides cores have been used to design (photo)catalysts as well as many other applications. Synthesis of all NDIs started from commercially available naphthalene-1,4,5,8-tetracarboxylic acid dianhydride (NDA) and the corresponding amine using reported procedure (see SI for details). We decided to work with a family of simple naphthalene diimides with different substitutions (NDI1-5 Figure 2). It is known that substitution in positions 2-, 3-, 6-, or 7- ("bay" substitution) with heteroatom confer to NDIs a strong absorption in the visible region allowing us to have a family of organic compounds with different spectra (Figure 3. and S1). Selection of cyclohexylamine for "bay" substitution group was done according to the reported improved stability of radical ions of related NDI bearing this group. ${ }^{35}$<smiles>[R]N1C(=O)c2ccc3c4c(ccc(c24)C1=O)C(=O)N([R])C3=O</smiles>

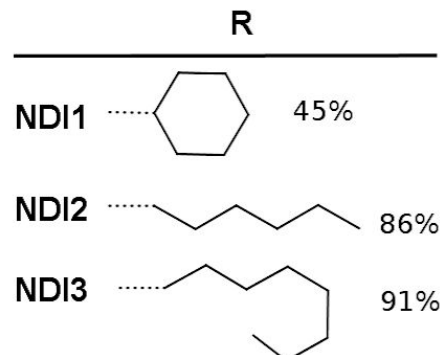

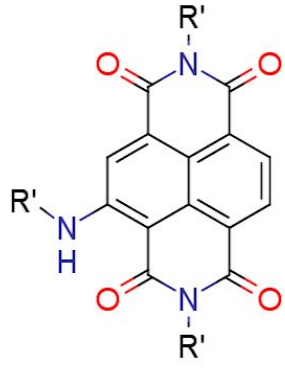

NDI4<smiles>[R]Nc1cc2c3c(c(N[R])cc4c3c1C(=O)N([R])C(=O)C4=O)C(=O)N([R])C2=O</smiles>

NDI5 $10 \%$

Figure 2. Structures and total yields of naphthalene diimides used in this work. 



\section{Photocatalyzed C-H homolytic aromatic substitution - Consecutive PET (conPET) process}

We began our investigation with a synthetically important $\mathrm{C}-\mathrm{C}$ bond-formation reaction using an aryl halide as starting material and visible-light irradiation. Typical conditions for a conPET reaction were first analysed using all NDI1-5 as catalysts. A mixture of 4-Bromobenzonitrile (as radical precursor), benzene (as radical acceptor) and $N, N$-Diisopropylethylamine (DIPEA) (sacrificial electron donor) was used as a model system working in different solvents and a blue light-emitting diode (LED) as source of visible light irradiation (Table 1 and SI).

Table 1. Photostimulated reactions catalysed by NDI1-5

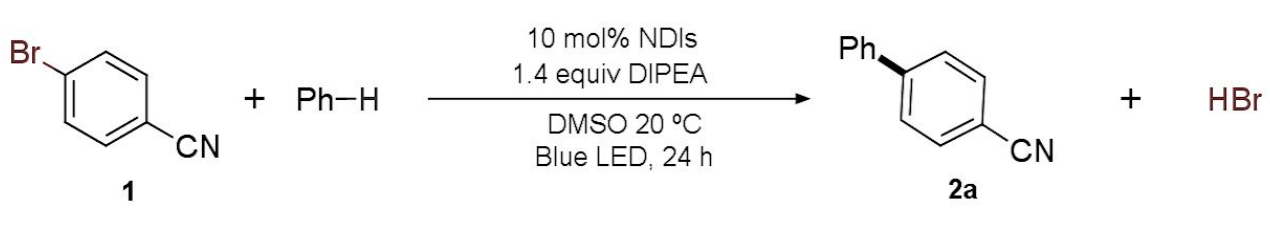

\begin{tabular}{|c|c|c|c|c|c|}
\hline \multirow{2}{*}{ Entry } & \multirow{2}{*}{ Catalyst } & \multirow{2}{*}{ Conv $^{b}$} & \multicolumn{3}{|c|}{ Products yield $(\%)^{c}$} \\
\hline & & & $2 a$ & $\mathrm{PhCN}$ & $\mathrm{MB}^{d}$ \\
\hline 1 & NDI1 & 30 & 18 & 12 & 100 \\
\hline 2 & NDI2 & 62 & 16 & 17 & 71 \\
\hline 3 & NDI3 & 60 & 18 & 20 & 78 \\
\hline 4 & NDI4 & 82 & 17 & 28 & 63 \\
\hline 5 & NDI5 & 66 & 16 & 21 & 71 \\
\hline $6^{e}$ & NDI1 & 16 & - & - & 84 \\
\hline$\overline{7^{f}}$ & NDI1 & 12 & $<1$ & $<1$ & 88 \\
\hline 8 & - & 0 & $<1$ & $<1$ & 100 \\
\hline \multicolumn{6}{|c|}{$\begin{array}{l}{ }^{a} \text { The photostimulated reaction was carried out under } \mathrm{N}_{2} \\
\text { atmosphere using BrPhCN }(0.1 \mathrm{mmol}) \text {, DIPEA }(1.4 \text { equiv.), } \\
1.1 \mathrm{~mL} \text { DMSO }([1]=0.059 \mathrm{M}) \text {, benzene }(65 \text { equiv., } 600 \mathrm{uL}) \text {, } \\
\text { and a NDI catalyst. Irradiation was carried out with Blue-LED } \\
(3 \mathrm{~W}) .{ }^{b} \text { The conversion (conv) was determined by } \\
\text { quantification of the recovered substrate. }{ }^{c} \text { Yields were } \\
\text { determined by HPLC (external standard method) and GC } \\
\text { (internal standard method). }{ }^{d} \text { Mass Balance. }{ }^{e} \text { Reaction in the } \\
\text { dark. }{ }^{f} \text { Reaction without DIPEA. }\end{array}$} \\
\hline
\end{tabular}

Results in Table 1 indicate that all NDIs promote the cross-coupling transformation. The efficiency of the catalysts (in terms of product formation) is similar and does not correlate with the overlap of their UV-Vis spectra and the source of irradiation. However, substrate consumption shows different values pointing out to a difference in the extent of side reactions with each catalyst. In all cases, development of highly absorbing by-products could be responsible for the incomplete conversion ("self-filter" effect). Since the main objective of the present study was a controlled comparison of two techniques no further optimization was performed. However, using $N$-methylpyrrole also gives the expected product an acceptable yield (see SI) and synthetic applications of NDIs are under investigation and will be published in a separate work.

Control experiments (Table 1 entries 6-8) confirmed that light, catalyst and electron donor (DIPEA) are all necessary for the reaction to occur. Reactions without trapping agent gave reduced product (benzonitrile, PhCN) and experiment with 1,1-diphenylethylene, a radical scavenger, gave the respective substitution product, supporting the generation of 4-cyanobenzyl radical (see SI). The presence of small amounts of water (100 equiv.) did not give any new product discoragin a cationic pathway. Analysis of the relations of coupling / reduced product are according to the expected reactivity of radicals with aromatic systems. ${ }^{36}$

When NDI1 is irradiated in the presence of DIPEA a new absorption in the visible region appears 

corresponding to the radical anion of NDI1 (NDI1--) as confirmed by spectro-electrochemical studies (see Figure 3) and are in agreement with the reported experiment of related NDIs derivatives. ${ }^{33,35}$ Signal for NDI • - reach a stationary state under continuous irradiation after $25 \mathrm{~min}$ and remain stable at least $60 \mathrm{~min}$ under these conditions (Figure 3). When irradiation is stopped (after $60 \mathrm{~min}$.) concentration of NDI1 •slowly decreases with the concomitant recovery of signals of neutral NDI1 (see inset Figure 3). This behaviour could be explained by back electron transfer and/or small leaks that allow a small diffusion of oxygen into the system. Being this as it be, these results support a reversible ET to NDI1 and low amount of degradation reaction between NDI1 - and the radical cation of DIPEA (or its derivatives). In addition, irradiation of NDI1 alone or in the presence of $\mathbf{1}$ did not affect the initial spectra, ruling out any PET between NDI1 and these compounds (See Figure in SI.); a result that is consistent with control experiments (Table 1 entry 7 ).
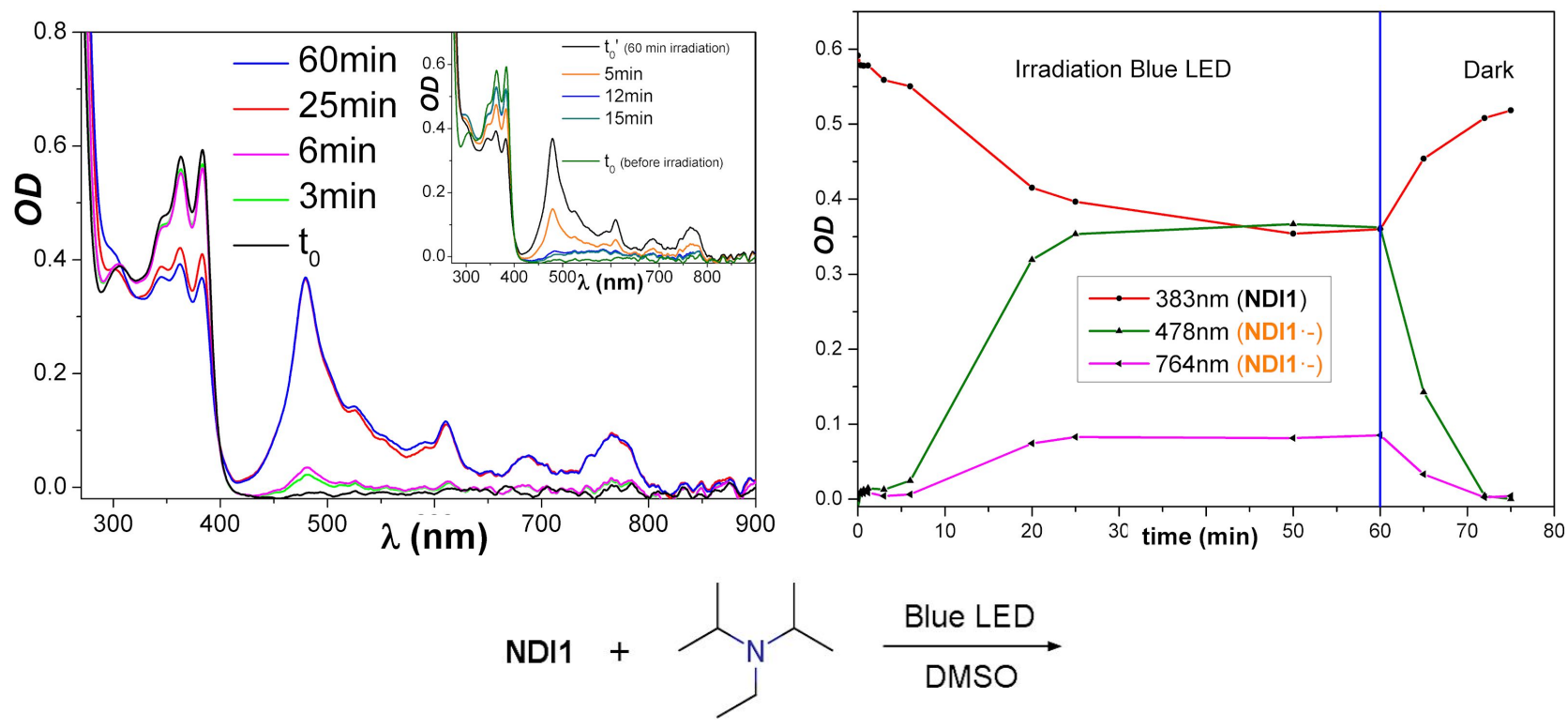

Figure 3. (a) Formation of radical anion of NDI1 in the presence of DIPEA. Inset: decay in the dark. (b) Time dependency of absorption at $\lambda_{\max }$ NDI1 and its radical anion.

When a mixture of NDI1, DIPEA and $\mathbf{1}$ is irradiated initial formation (5 min.) of NDI1 - is observed without a noticeable difference compared to the experiment in the absence of the aryl halide (Figure 4a.). However, since $10 \mathrm{~min}$ of irradiation a change in the spectra is observed suggesting the formation of new species. The NDI1 - - signal instead of reaching a stationary point, starts to decrease and a noticeable bleaching of the catalyst after $25 \mathrm{~min}$ is observed (Figure 4a and SI). However, if irradiation is stopped after 3 min and the resulting mixture is keep in the dark, NDI1 - behaviour resembles the one obtained in the absence of $\mathbf{1}$ (Figure 4a inset). Irradiation of a reaction mixture (NDI1 + DIPEA + $\mathbf{1}+$ benzene) also result in the appearance of the characteristic absorption of the NDI1 - - although a slower kinetic of formation and degradation are observed (Figure $4 \mathrm{~b}$ and SI).

It is important to mention that characteristic absorption of dianion of NDI1 (see below) was never detected in any experiment. 


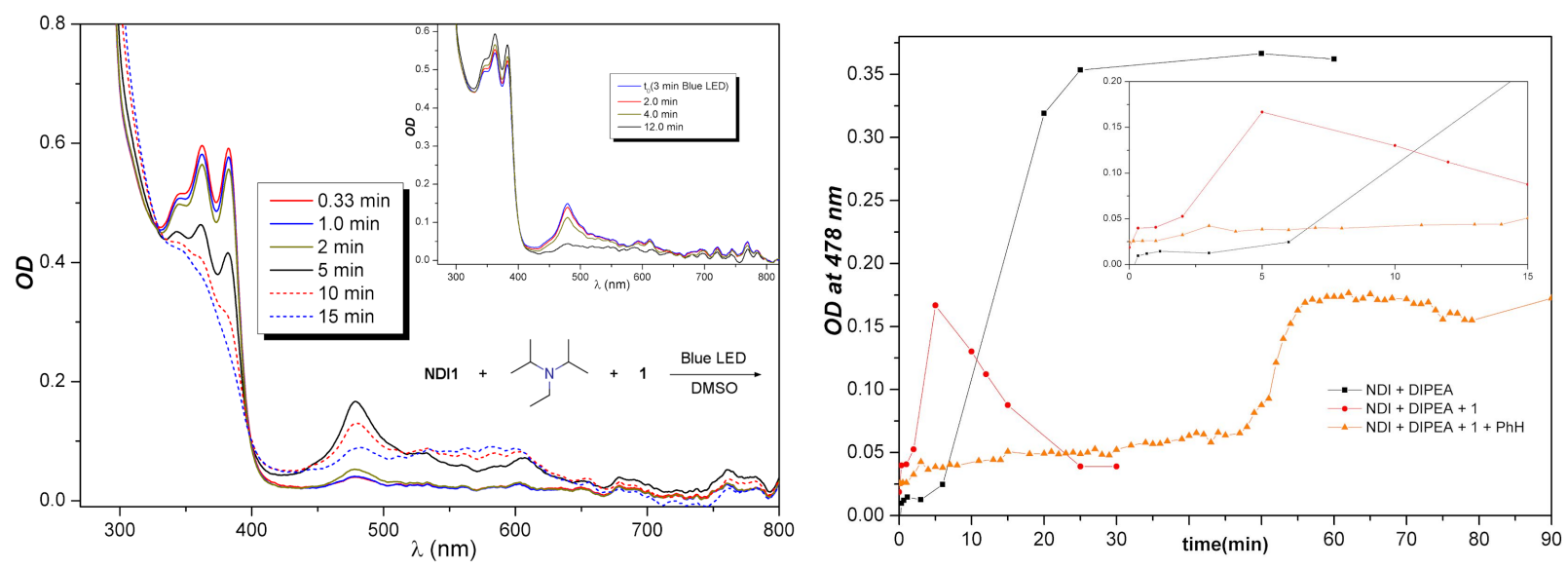

Figure 4. Irradiation of NDI1 in mixtures with DIPEA, 1 and benzene. (a) NDI1 + DIPEA + 1. Inset: decay in the dark after 3 min. of irradiation. (b) Changes at $478 \mathrm{~nm}$ vs. time: NDI1 + DIPEA (black), NDI1 + DIPEA + 1 (red) and NDI1 + DIPEA + benzene (orange)

\section{Electro-photocatalyzed C-H homolytic aromatic substitution}

The transformation indicated in equation 1 was also studied in an electro-photocatalyzed experiment. We anticipated that the key NDI1 - could be generated in the cathode avoiding the need for a sacrificial electron donor such as DIPEA and this radical anion fuel with redox energy could participate in a PET process after irradiation with visible-light (see Scheme 5.).

The electrochemical properties of NDI1-4 determined by cyclic voltammetric (CV) experiments show two quasi-reversible cathodics peaks associated with the $\mathrm{NDI} / \mathrm{NDI} \bullet-$ and $\mathrm{NDI} \bullet-/ \mathrm{NDI}^{2-}$ processes with the current being slightly smaller during the reduction scan. (Table 2). ${ }^{37}$ Thus, the radical anion and dianion formed display good stability on the time scale of the CV measurement. These results are in agreement with reported studies. Spectroelectrochemical analysis give the UV-Vis spectra of NDI•- and NDI $^{2-}$ showing all studied NDIs strong absorption in the visible region (Figure 5 and Figures in SI).

Table 2. Electrochemical properties of NDIs used in this study (values in V vs. SCE).

\begin{tabular}{ccc}
\hline $\mathbf{P C}$ & $\mathbf{E}^{\mathbf{0}}$ (NDI/NDI') & $\mathbf{E}^{\circ}$ (NDI $^{\circ} / \mathbf{N D I}^{\mathbf{2}}$ ) \\
\hline NDI1 & -0.45 & -0.91 \\
NDI2 & -0.46 & -0.85 \\
NDI3 & -0.50 & -0.91 \\
NDI4 & -0.62 & -1.02 \\
\hline
\end{tabular}
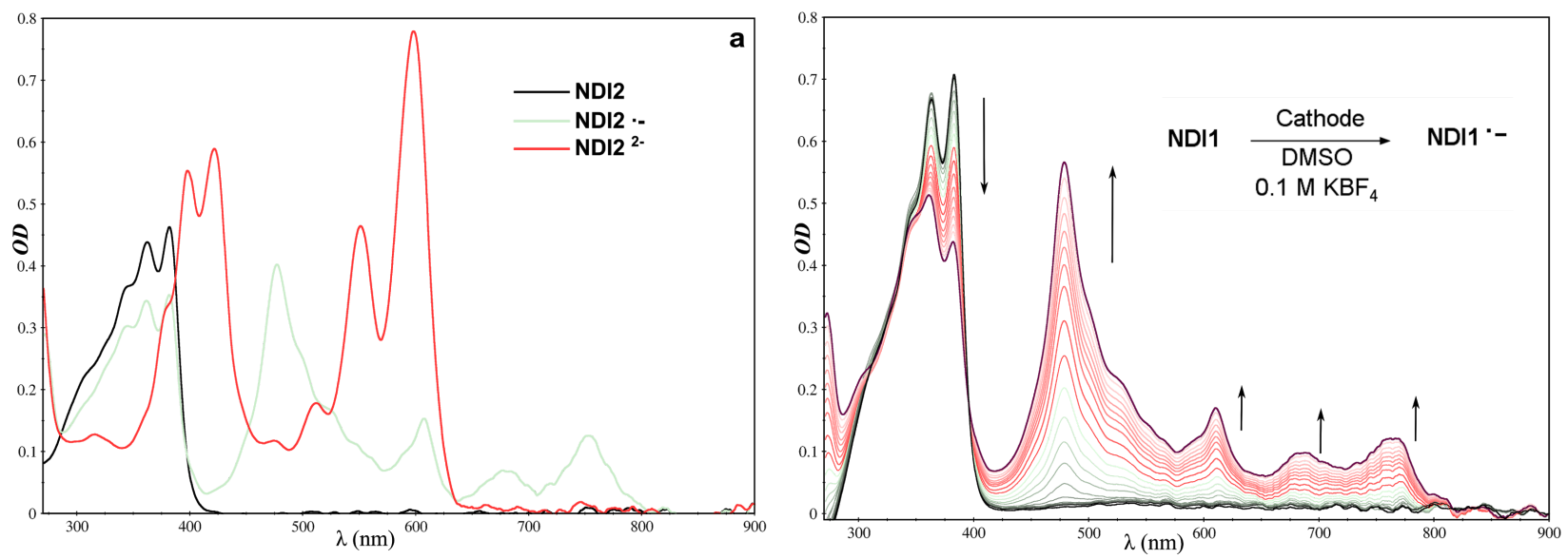

Figure 5. Spectro-electrochemical measurement of NDI. (a) Spectroelectrochemical potential scan experiment of NDI2 in a $1 \mathrm{~mm}$ cuvette. (b) UV-Vis spectra at constant current electrolysis of NDI1 in a $10 \mathrm{~mm}$ cuvette.

The electrolysis of a solution of NDI1-3 give a mixture of NDI1/NDI1 - - working at constant current and the presence of dianion was never detected in this condition (Figure 5b and SI). The evolution was followed by in-line measurement of UV-Vis spectra provided a $10 \mathrm{~mm}$ UV cuvette was used as the cathodic cell (see SI). Based on these positive results, we studied the electro-photocatalytic C-H substitution reaction of equation 2 in a divided cell photo-reactor (see SI). Simultaneous visible light irradiation (blue LED) and electrolysis of a mixture composed by catalytic NDI2, 1 and the aromatic radical-trap (benzene) gave the expected C-H substitution product in several electrochemical conditions (Table 3).

Table 3. Electro-photostimulated reactions catalysed by NDI2 $^{\mathrm{a}}$

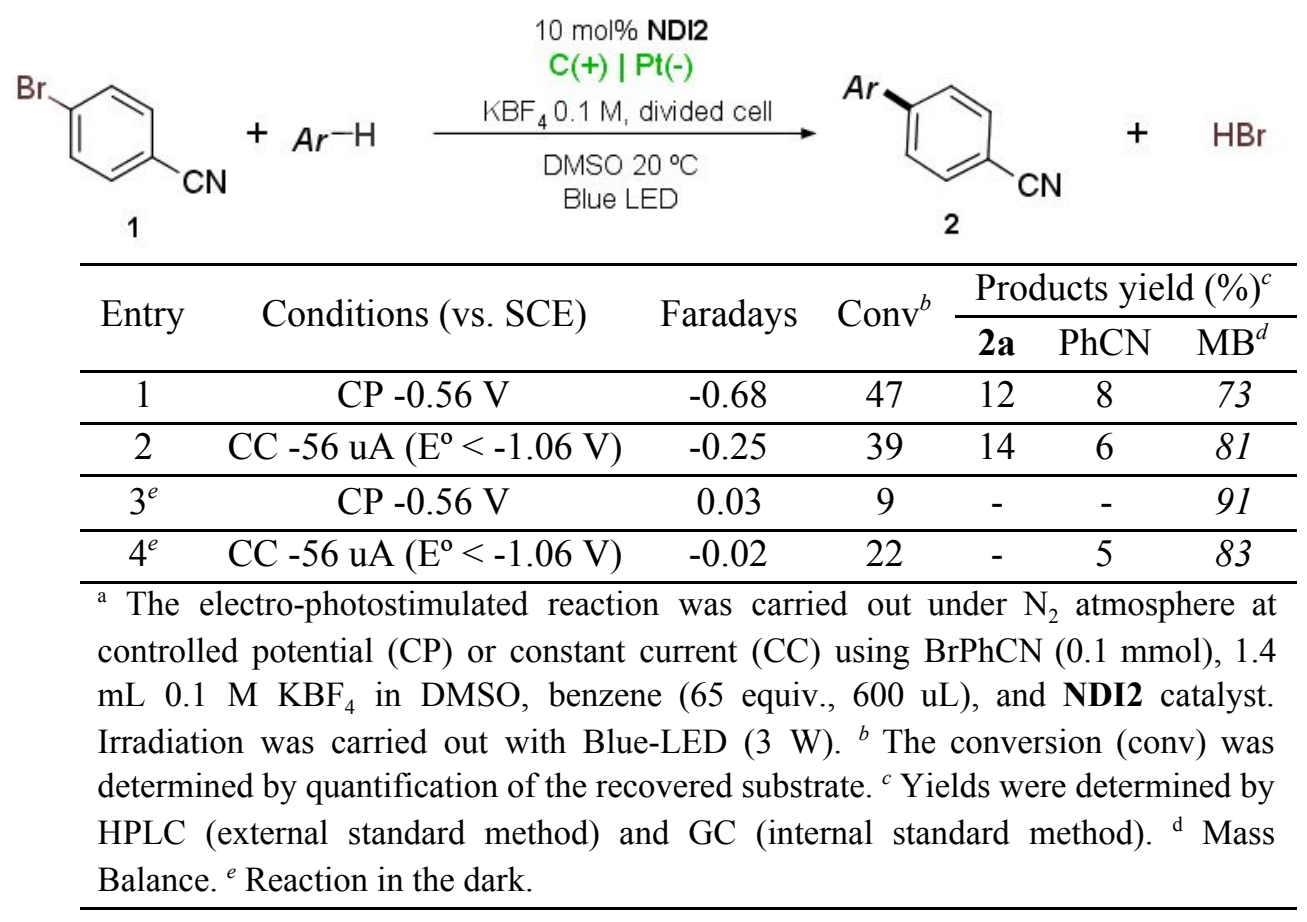

Control experiments (Table 3 and SI) confirmed that light, catalyst and electricity are all necessary for the reaction to occur. Reactions without radical-trap gave the reduced product $(\mathrm{PhCN})$.

The use of inorganic supporting electrolyte $\left(\mathrm{KBF}_{4}\right)$ was deliberately chosen in order to avoid ammonium salts which estresults in a real replacement of amines in the present system as compared to the conPET approach.

To gain a deeper understanding of catalytic transformation an electrolytic solution of NDI1-3•- was prepared and its evolution followed by UV-Vis under different stimulus in the absence of current (Figures 6-7 and SI); detailed experiments for NDI3 follows. After electrolysis of a saturated solution of NDI3 is stopped, a solution of NDI3- is stable showing small changes at dark or under irradiation with a blue LED (Figure 6 and SI). ${ }^{38}$ When 1 is added to an electrogenerated solution of NDI3•- (under $\mathrm{N}_{2}$ ) all characteristic peaks corresponding to the NDI3-- remain for $30 \mathrm{~min}$ in the dark, showing a behavior resembling a pure solution of NDI3- (Figure 7 and SI). However, irradiation with a blue LED led to the total consumption of the NDI3 - within 10 min supporting a PET process from NDI3 - to the aryl bromide (Figure 8). ${ }^{39}$ Electrolysis of the resulted solution in the absence of irradiation regenerates the NDI3•- signals which are again consumed by irradiation (see SI). Repetition of this cycle finally results in the bleaching of the catalyst in a similar way as observed for the photo-generated radical anion in the presence of DIPEA (Figure 4). 

Experiments with the reaction mixture of eq. 2 with benzene show similar behaviour indicating that PET to 1 is not affected by the presence of the radical-trap. However, changes in the initial spectra are clear in the "reaction mixture" in line with its higher complexity, pointing to the formation of charge transfer complexes between NDI3--, 1 and benzene. Another interesting point is the higher persistence of the signal at $475 \mathrm{~nm}$ when the trapping agent is present, especially after $5 \mathrm{~min}$ of irradiation (compare Figures 7 and 8). This could indicate some recovery of the radical anion which is consistent with a catalytic cycle not possible in the absence of radical-trap.

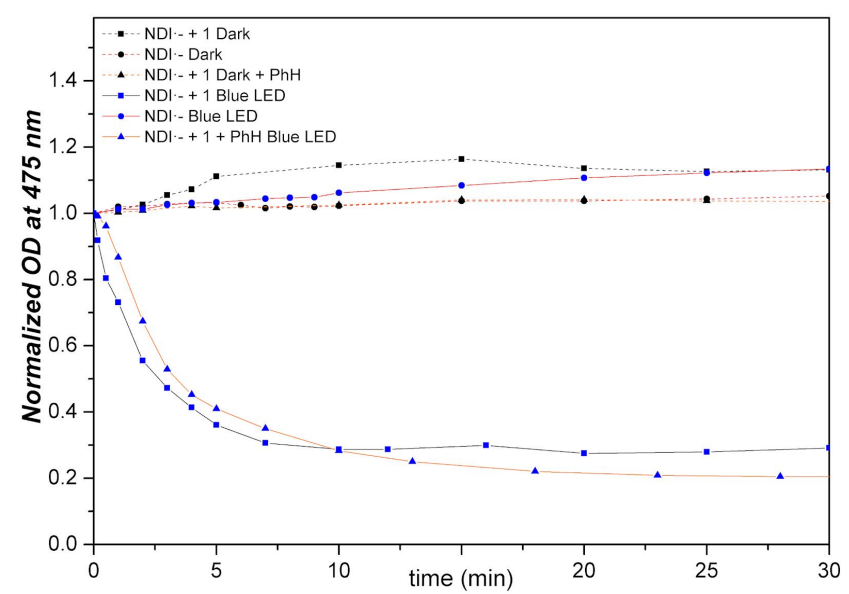

Figure 6. Irradiation of electrochemically prepared NDI1 - and mixtures with $\mathbf{1}$ and benzene. Changes at key $\lambda$ vs. time.
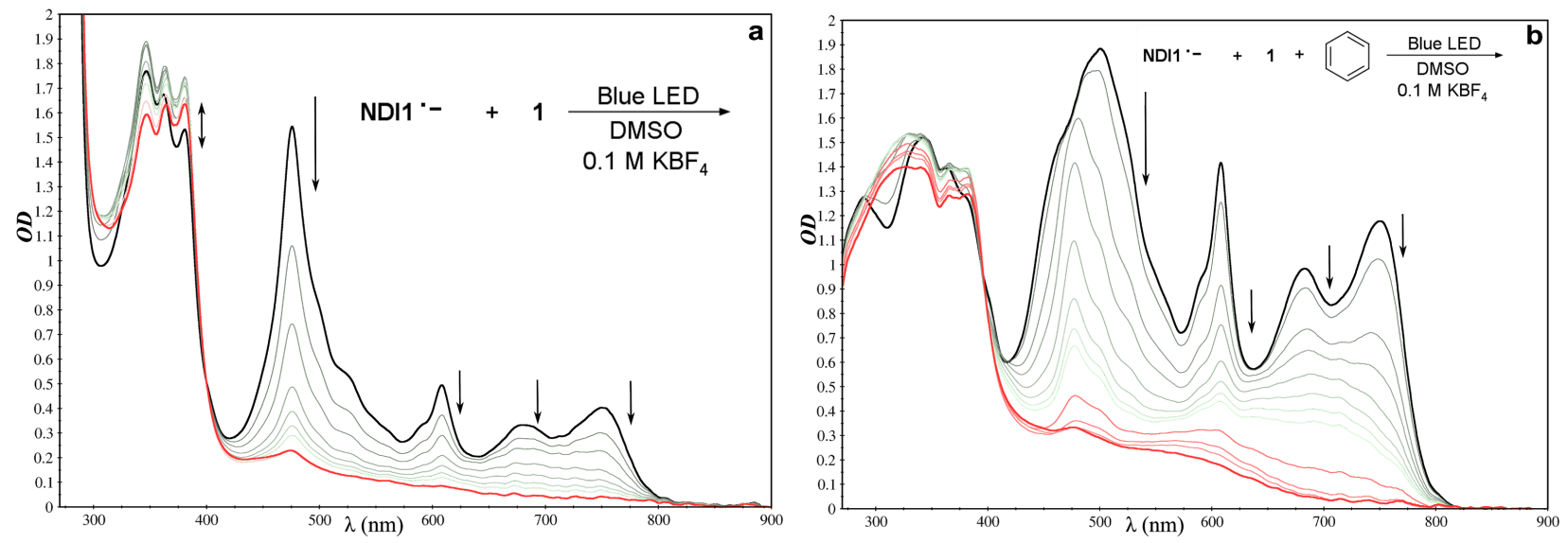

Figure 7. Irradiation of a mixture electrochemically prepared NDI3•- with (a) $\mathbf{1}$ and (b) $\mathbf{1}+$ benzene . From black-gray $(0,10 \mathrm{~s}, 30 \mathrm{~s}, 1 \mathrm{~min}, 2 \mathrm{~min}, 3 \mathrm{~min}, 4 \mathrm{~min})$ to red $(5 \mathrm{~min}, 10 \mathrm{~min}, 15 \mathrm{~min}, 30 \mathrm{~min}, 48$ $\min )$.

Similar experiments with NDI1 and NDI2 demonstrate the generality of the observed behaviour (see SI).

Mechanism of electro-photocatalysis and conPET catalysis.

Based on the previous experiments, we propose an overall mechanism for both photoinduced transformations as depicted in Scheme 5. 



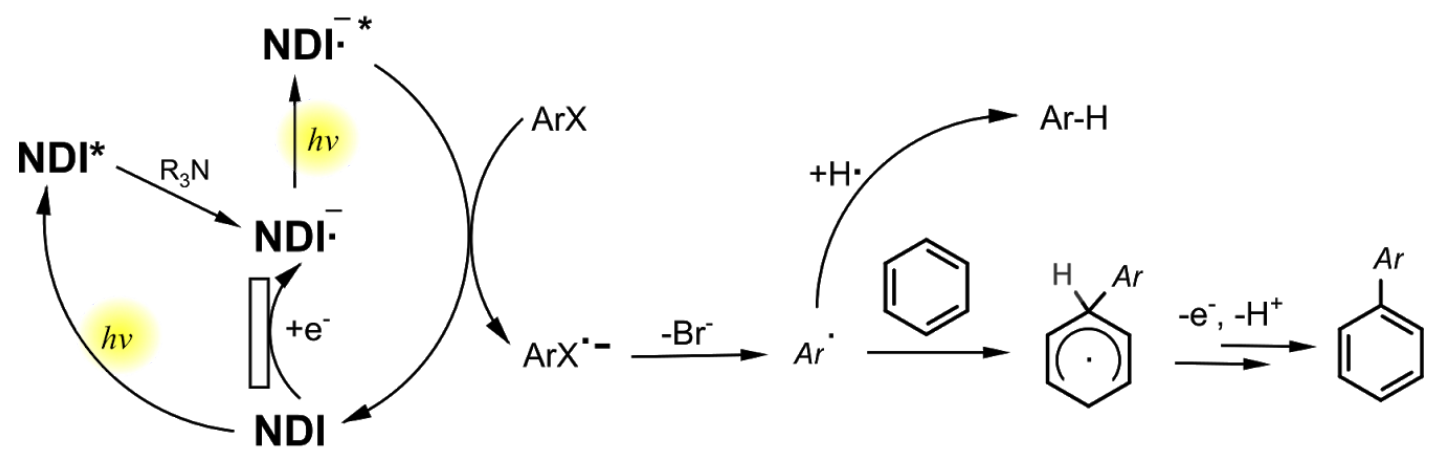

Scheme 5. Proposed mechanism

Irradiation with blue LED of NDIs in the presence of DIPEA generates the NDI•- in a stable and measurable concentration. This fact not only probes a PET from DIPEA to NDI* but also confirms that back ET is slow enough to allow a stable concentration of radical anion, well above typical reactive intermediates, which is a requirement for an efficient excitation. On the other hand, electrochemical generation of NDI•- probes the possibility of having an even more stable solution of NDI•- (compare decay times in SI) by avoiding back ET from a radical cation (or a derivative) of the electron donor. All together our results show a clear possibility of generating stable solutions (under $\mathrm{N}_{2}$ ) of radical anions of naphthalene diimides giving a strong base for the occurrence of photostimulated reactions from these species. Another important point of these set of photochemical and electrochemical experiments is the absence of dianions of NDI, a situation that is expected giving the presence of neutral NDI at all times in these "analytical" experiments as well as in the preparative ones, that will quench any dianion (if formed) to a pair of radical anions.

Following the reactivity of NDI•-, it is clear the occurrence of a photoinduced reaction with $\mathbf{1}$ (and the absence of reaction in the dark). Besides, a PET reaction is confirmed based on the formation of aryl radicals, as probed by the product distributions and the recovery of neutral NDI. However, it is worth to mention that the specific mechanism of this PET could be more complex than a typical dynamic quenching of NDI•-, especially in the presence of large amounts of third aromatic species such as benzene.

After key ET to the aryl halide fast fragmentation follows generating the reactive aryl radical. Initial fate of the aryl radical formed is well established in the actual experimental conditions and includes the abstraction of $\mathrm{H}$ from the solvent (reduced by-product) and homolytic aromatic substitution (HAS) to obtain the substitution product. Several mechanisms are possible for a HAS reaction with difference in the path of rearomatization of radical I (Scheme 5), including an $\mathrm{H}$ abstraction or a two-steps path with ET and proton elimination or vice versa. It has been demonstrated that initial proton elimination requires a strong base such as alkoxide anions and could be discarded in the present scenario. Although $\mathrm{H}$ abstraction from radical could not be ruled out, the present system is more consistent with an oxidation-proton elimination sequence with an ET to NDI, based on our spectroelectrochemical studies. This affirmation would imply an electrocatalytic process for the electro-phototransformation, however this could not be confirmed based on the consumption of charge probably due to undesired process. A similar situation is true for the conPET process since the electron donor is not consumed in an stoichiometric amount, however excess of donor is necessary to reach a steady-state in which the concentration of radical anion is suitable for the efficient excitation and a reasonable kinetic.

The two approaches analyzed have close related mechanisms and common problems appear such as catalysts bleaching and slow kinetic for the transformation. Initial comparison of the two approaches slightly favors the photochemical conPET process in terms of product yields and setup simplicity. However, the electro-photochemical transformation gave a better relation product/reduced-product; the known $\mathrm{H}$ donor capabilities of radical anions of amines could explain this observation. In addition, in the electro-PET 

conditions there are (potentially) more possibilities of control of the concentration of the key specie NDI•-, which could influence the kinetic of the reaction and yields. ${ }^{40}$ Besides it is worth mentioning that, in contrast to reported works, no sacrificial electrodes or donors have been used in the present work that result in a real replacement of amines as donor by the cathode. The addition of supporting electrolyte represents only 1-2 equiv. of a cheap non-toxic additive in comparison with 1.4 equiv. of toxic amines.

\section{Conclusion}

In the present work, we were able to clearly define the mechanism of a consecutive PET and electro PET transformation with the same system, identifying the radical anion of naphthalene diimides as the key intermediates.

Both approaches are comparable in yields and kinetics and although a conPET process shows in principle a simpler setup the electro-PET process improves selectivity and gives more room for optimization. Having a process that could follow a conPET or an electro-PET, our results suggest that a particular analysis is still necessary in order to favour one approach for the synthesis of a target compound.

The present work offers a system that could be used for a more profound comparison in different labs and experimental setups which could include, for instance, the important factor of energy consumption.

\section{Acknowledgements}

This work was supported in part by Agencia Córdoba Ciencia, Consejo Nacional de Investigaciones Científicas y Técnicas (CONICET), Secretaría de Ciencia y Tecnología, Universidad Nacional de Córdoba (SECyT), Agencia Nacional de Promoción Científica y Técnica (ANPCyT) and the Alexander von Humboldt Foundation.

\section{Supporting Information}

General remarks, extra experimental details, UV-Vis spectra, time resolved spectra, ${ }^{1} \mathrm{H}$ NMR and ${ }^{13} \mathrm{C}$ NMR spectra for the products.

\section{References}

(1) Marzo, L.; Pagire, S. K.; Reiser, O.; König, B. Angew. Chemie - Int. Ed. 2018, 57, 10034-10072.

(2) Romero, N. A.; Nicewicz, D. A. Chem. Rev. 2016, 116, 10075-10166.

(3) Prier, C. K.; Rankic, D. a; MacMillan, D. W. C. Chem. Rev. 2013, 113, 5322-5363.

(4) Rossi, R.; Bardagi, J.; Buden, M. Curr. Org. Synth. 2017, 14, 398-429.

(5) Ghosh, I.; Marzo, L.; Das, A.; Shaikh, R.; König, B. Acc. Chem. Res. 2016, 49, 1566-1577.

(6) Little, R. D.; Moeller, K. D. Chem. Rev. 2018, 118, 4483-4484.

(7) Francke, R.; Little, R. D. Chem. Soc. Rev. 2014, 43, 2492-2521.

(8) Capaldo, L.; Quadri, L. L.; Ravelli, D. Angew. Chemie - Int. Ed. 2019, 58, 17508-17510.

(9) Barham, J. P.; König, B. Angew. Chemie - Int. Ed. 2019, 2-18.

(10) Ghosh, I.; Ghosh, T.; Bardagi, J. I.; Konig, B. Science (80-. ). 2014, 346, 725-728.

(11) Svanholm, U.; Parker, V. D. J. Am. Chem. Soc. 1972, 94, 5507-5508.

(12) Lund, H.; Carlsson, H. S. Acta Chem. Scand. 1978, 32b, 505-509.

(13) Bardagi, J. I.; Ghosh, I. In Visible Light-Active Photocatalysis; Ghosh, S., Ed.; Wiley-VCH Verlag GmbH \& Co. KGaA: Weinheim, Germany, 2018; pp 75-114. 

(14) Ghosh, I. Phys. Sci. Rev. 2019, 4, 1-11.

(15) Kim, H.; Kim, H.; Lambert, T. H.; Lin, S. J. Am. Chem. Soc. 2020, 142, 2087-2092.

(16) Cowper, N. G. W.; Chernowsky, C. P.; Williams, O. P.; Wickens, Z. K. J. Am. Chem. Soc. 2020, 142, 2093-2099.

(17) Gong, H. X.; Cao, Z.; Li, M. H.; Liao, S. H.; Lin, M. J. Org. Chem. Front. 2018, 5, 2296-2302.

(18) Shang, J.; Tang, H.; Ji, H.; Ma, W.; Chen, C.; Zhao, J. Cuihua Xuebao/Chinese J. Catal. 2017, 38, 2094-2101.

(19) Zeng, L.; Liu, T.; He, C.; Shi, D.; Zhang, F.; Duan, C. J. Am. Chem. Soc. 2016, 138, 3958-3961.

(20) Rosso, C.; Filippini, G.; Cozzi, P. G.; Gualandi, A.; Prato, M. ChemPhotoChem 2019, 3, $193-197$.

(21) Zeman, C. J.; Kim, S.; Zhang, F.; Schanze, K. S. J. Am. Chem. Soc. 2020, jacs.9b13027.

(22) Ghosh, I.; König, B. Angew. Chemie Int. Ed. 2016, 55, 7676-7679.

(23) Nelleborg, P.; Lund, H.; Eriksen, J. Tetrahedron Lett. 1985, 26, 1773-1776.

(24) Bardagi, J. I.; Ghosh, I.; Schmalzbauer, M.; Ghosh, T.; König, B. European J. Org. Chem. 2018, 2018, 34-40.

(25) Robertson, P. K. J.; Eggins, B. R. J. Chem. Soc. Perkin Trans. 2 1994, No. 8, 1829.

(26) Shukla, S. S.; Rusling, J. F. J. Phys. Chem. 1985, 89, 3353-3358.

(27) Compton, R. G.; Eklund, J. C.; Fisher, A. C.; Waller, A. M. J. Chem. Soc. Faraday Trans. 1990, 86, 2951-2953.

(28) Costentin, C.; Fortage, J.; Collomb, M. N. J. Phys. Chem. Lett. 2020, 11, 6097-6104.

(29) Rombach, D.; Wagenknecht, H. A. ChemCatChem 2018, 10, 2955-2961.

(30) Cole, J. P.; Chen, D. F.; Kudisch, M.; Pearson, R. M.; Lim, C. H.; Miyake, G. M. J. Am. Chem. Soc. 2020, 142, 13573-13581.

(31) Niu, L.; Jiang, C.; Liang, Y.; Liu, D.; Bu, F.; Shi, R.; Chen, H.; Chowdhury, A. D.; Lei, A. J. Am. Chem. Soc. 2020, 142, 17693-17702.

(32) Kobaisi, M. Al; Bhosale, S. V. S. V.; Latham, K.; Raynor, A. M.; Bhosale, S. V. S. V. Chem. Rev. 2016, 116, 11685-11796.

(33) Fujitsuka, M.; Kim, S. S.; Lu, C.; Tojo, S.; Majima, T. J. Phys. Chem. B 2015, 119, 7275-7282.

(34) He, J.; Li, J.; Han, Q.; Si, C.; Niu, G.; Li, M.; Wang, J.; Niu, J. ACS Appl. Mater. Interfaces 2020, 12, 2199-2206.

(35) Ajayakumar, M. R.; Asthana, D.; Mukhopadhyay, P. Org. Lett. 2012, 14, 4822-4825.

(36) Faster addition of aryl radicals to pyrrole compared to benzene has been reported.

(37) NDI5 could not be measured due to poor solubility of the compound in DMSO.

(38) In all cases a mixture of RA and neutral compound is obtained. Small changes are detected in the dark and irradiated solution resulting, in all cases studies, in an small increase of the signals corresponding to the RA without a change on the topology of the spectra.

(39) It is known that radical anions of aromatic halides suffer a fast $\left(10^{9-11} \mathrm{~s}^{-1}\right)$ fragmentation generating species with negligible absorptivity in the measured spectra. 

(40) Explorations of these "solution" are under investigation and will be published in a separate work 
